\title{
Nonmetropolitan Counties Have a Standard Definition: Response to Hughey et al
}

\author{
Andrea Wendling, MD | Julie Phillips, MD, MPH \\ Published: 1/10/2018 | DOI: 10.22454/PRiMER.2017.116030
}

To the Editor,

We would like to raise a concern about a recent article published in PRiMER: "Risk for Unintended Pregnancy Among Latino Men and Women in a Nonmetropolitan County in Michigan."1 Dr Hughey et al studied the risk of unintended pregnancy in Latino men and women in a setting they described as a nonmetropolitan county in Michigan. In the background section, the study was contrasted with previously published studies of metropolitan regions. Their findings showed a higher risk for unintended pregnancy in the study sample than that seen nationally in this population. In the discussion, they hypothesized that this discrepancy may be due to Latinos in nonmetropolitan communities lacking the resources available to those in metropolitan communities, including health-related resources such as language-concordant health care providers and facilities, lower neighborhood social cohesion, or fewer culturally appropriate community resources.

Our major concern with this study is that Washtenaw County is not a nonmetropolitan county. ${ }^{2}$ Washtenaw county is the central county of the Ann Arbor, Michigan metropolitan statistical area, and includes the principal city of Ann Arbor (population 113,934), home of the University of Michigan Health System. ${ }^{3}$ The Office of Management and Budget considers Washtenaw county metropolitan ${ }^{4}$; the US Department of Agriculture has assigned a Rural-Urban Continuum Code of 2 to Washtenaw County-a metropolitan designation ${ }^{5}$; and the US Census Bureau defines Washtenaw County as a "mostly urban" county, the most urban classification available. ${ }^{6}$

This of course does not mean that every area within Washtenaw county is urban, as on a county basis the definitions above do include some rurality. The US Census Bureau definition of "mostly urban," for instance, includes all counties where over $50 \%$ of the population resides in an urban community. Washtenaw county is $16 \%$ rural. However, this small amount of rurality does not make the county nonmetropolitan. And although the authors may have assumed that most of the Latino population resided within the $16 \%$ of the county considered rural, this does not bear true when examining census data, which shows the highest numbers of residents of Latino origin in the eastern urban portions of the county, rather than the western rural portions. ${ }^{7}$

Although it is interesting that the population studied here was at higher risk of unintended pregnancy, we do not feel it represents a nonmetropolitan group. Accurate and specific descriptions of the population studied, and the resources available to them, are important. Although the authors do state that the results may not be generalizable, the role of descriptive research is to find information that can be applied to the larger world. Based on the inaccurate nonmetropolitan description, readers may reasonably but inappropriately conclude that the authors have uncovered a phenomenon that may also be true in actual nonmetropolitan communities. Moreover, in describing their population as nonmetropolitan, the authors are losing an opportunity to explore alternative explanations for their findings.

We suggest that the community studied should not be represented as nonmetropolitan, unless the authors are able to more completely justify this assertion in a scholarly manner, either with an alternate county-level designation of nonmetropolitan or on a community level, by documentation of exclusion of those persons of Latino origin that resided within the urban portions of this county. 


\section{Corresponding Author}

Andrea Wendling, MD

965 Fee Rd., East Lansing, MI 48824. 231-675-2245

wendli14@msu.edu

\section{Author Affiliations}

Andrea Wendling, MD - Michigan State University College of Human Medicine, East Lansing, MI

Julie Phillips, MD, MPH - Michigan State University College of Human Medicine, Sparrow Family Medicine Residency Program

\section{References}

1. Hughey KL, Llanes M, Plegue M, Kruger DJ, Waller A, Ledón C, Chang T. Risk for unintended pregnancy among latino men and women in a nonmetropolitan county in Michigan. PRiMER. 2017; 1:21. https://doi.org/10.22454/PRiMER.2017.651218.

2. United States Census Bureau. 2010 Office of Management and Budget (OMB) Standards, Metropolitan and Micropolitan. https://www.census.gov/programs-surveys/metro-micro/about.html. Revised January 11, 2017. Accessed November 6, 2017.

3. United States Census Bureau. American Fact Finder, Annual Estimates of the Resident Population, Ann Arbor, Michigan. https://factfinder.census.gov/bkmk/cf/1.0/en/place/Ann Arbor city, Michigan/POPULATION /DECENNIAL_CNT. Accessed December 7, 2017.

4. United States Census Bureau. Geographies, Delineation Files of Core Based Stastical Areas, Metropolitan Divisions, and Combined Statistical Areas. https://www.census.gov/geographies/reference-files/time-series /demo/metro-micro/delineation-files.html. Accessed November 6, 2017.

5. United States Department of Agriculture Economic Research Service. Rural-Urban Continuum Codes. https://www.ers.usda.gov/data-products/rural-urban-continuum-codes/. Accessed November 6, 2017.

6. United States Census Bureau. Geography, County Classification Lookup Table. http://www2.census.gov /geo/docs/reference/ua/County_Rural_Lookup.xlsx. Accessed December 7, 2017.

7. United States Census Bureau, American Fact Finder, Race and Hispanic or Latino: 2010 - County -- County Subdivision and Place 2010 Census Summary File 1, Washtenaw County. https://factfinder.census.gov /bkmk/table/1.0/en/DEC/10_SF1/QTP3/0500000US26161. Accessed December 7, 2017.

Copyright $@ 2018$ by the Society of Teachers of Family Medicine

Related Content

Risk for Unintended Pregnancy Among Latino Men and Women in a Nonmetropolitan County in Michigan 